\title{
Biodegradable magnesium-based implants in bone studied by synchrotron radiation microtomography
}

Julian Moosmann

Berit Zeller-Plumhoff

D. C. Florian Wieland

Silvia Galli

Diana Krüger

Thomas Dose

Hilmar Burmester

Fabian Wilde

Martin Bech

Niccolò Peruzzi

Björn Wiese

Alexander Hipp

Felix Beckmann

Jörg Hammel

Regine Willumeit-Römer 


\title{
Biodegradable magnesium-based implants in bone studied by synchrotron radiation microtomography
}

\author{
Julian Moosmann*a $^{\mathrm{a}}$, Berit Zeller-Plumhoff ${ }^{\mathrm{a}}$, D. C. Florian Wieland ${ }^{\mathrm{a}}$, Silvia Gallib ${ }^{\mathrm{b}}$, Diana Krüger ${ }^{\mathrm{a}}$, \\ Thomas Dose ${ }^{\mathrm{a}}$, Hilmar Burmester ${ }^{\mathrm{a}}$, Fabian Wilde ${ }^{\mathrm{a}}$, Martin Bech ${ }^{\mathrm{c}}$, Niccolò Peruzzi ${ }^{\mathrm{c}}$, Björn Wiese $^{\mathrm{a}}$, \\ Alexander Hipp ${ }^{\mathrm{a}}$, Felix Beckmann ${ }^{\mathrm{a}}$, Jörg Hammel ${ }^{\mathrm{a}}$, Regine Willumeit-Römer ${ }^{\mathrm{a}, \mathrm{d}}$ \\ ${ }^{\mathrm{a} I n s t i t u t e}$ of Materials Research, Helmholtz-Zentrum Geesthacht, Max-Planck-Str. 1, D-21502 \\ Geesthacht, Germany; \\ ${ }^{\mathrm{b}}$ Faculty of Odontology, Malmö University, 20506 Malmö, Sweden; \\ ${ }^{\mathrm{c} C l i n i c a l}$ Sciences, Lund University, 22185 Lund, Sweden; \\ ${ }^{\mathrm{d}}$ Institute of Materials Science, Christian-Albrechts-Universität zu Kiel, Christian-Albrechts-Platz 4, \\ D-24118 Kiel, Germany; \\ *corresponding author: julian.moosmann@hzg.de; phone +49 40 8998-6904; www.hzg.de
}

\begin{abstract}
Permanent implants made of titanium or its alloys are the gold standard in many orthopedic and traumatological applications due to their good biocompatibility and mechanical properties. However, a second surgical intervention is required for this kind of implants as they have to be removed in the case of children that are still growing or on patient's demand. Therefore, magnesium-based implants are considered for medical applications as they are degraded under physiological conditions. The major challenge is tailoring the degradation in a manner that is suitable for a biological environment and such that stabilization of the bone is provided for a controlled period. In order to understand failure mechanisms of magnesium-based implants in orthopedic applications and, further, to better understand the osseointegration, screw implants in bone are studied under mechanical load by means of a push-out device installed at the imaging beamline P05 of PETRA III at DESY. Conventional absorption contrast microtomography and phasecontrast techniques are applied in order to monitor the bone-to-implant interface under increasing load conditions. In this proof-of-concept study, first results from an in situ push-out experiment are presented.
\end{abstract}

Keywords: Magnesium alloys, biodegradation, biodegradable metallic implants, bone-implant-interface, bone healing, $\mathrm{X}$-ray tomography, synchrotron radiation, microCT, in situ mechanical testing, load frame

\section{INTRODUCTION}

Since the 1950s, permanent metallic implants made of Titanium (Ti) or its alloys have been widely used in medical applications because of their biocompatibility and mechanical properties ${ }^{1,2}$. In orthopedic, cranio-facial, dental or trauma surgery they are the de facto gold standard because they integrate well into the bone. However, in pediatric applications where children are still growing, on patient's demand, or in rare cases of allergic reactions to Ti or metallic sensitivity, Ti-based implants need to be removed in a second surgical intervention. This results in an increased of risk of morbidity for the patients and higher healthcare costs. Implants made of biodegradable polymers are associated with unwanted side effects such as bone resorption (osteolysis), an overly long integration time in the bone, and a limited loadbearing capability. Here, $\mathrm{Mg}$ and its alloys have gained much interest in medical applications due to their biocompatibility, biodegradability $^{3}$, and mechanical properties. Magnesium-based materials have sufficient initial strength for load-bearing applications and degrade under physiological conditions into products well-tolerated by the body. The Young's modulus of Mg-based implants is very similar to bone which reduces the risk of stress shielding and subsequent bone atrophy. Thus, using biodegradable Mg-based implants, a second surgical intervention to remove the implant after bone healing can be avoided. However, fast or uncontrolled corrosion is associated with strong hydrogen and ion release and severe $\mathrm{pH}$ changes which can lead to a loss of mechanical stability and undesirable biological reactions ${ }^{4}$. A major challenge is

Developments in X-Ray Tomography XI, edited by Bert Müller, Ge Wang, Proc. of SPIE Vol. 10391, $1039100 \cdot$ (c) 2017 SPIE · CCC code: 0277-786X/17/\$18 · doi: 10.1117/12.2275121 
tailoring the degradation in a manner that ensures good osseointegration which results in the formation of a tight junction between implant and bone. For this, knowledge on the bone-to-implant interface is crucial as it determines the mechanical strength and governs the failure of the bone to implant connection. However, its formation and properties are yet poorly understood as the in vivo degradation behavior is difficult to predict due to the complex interactions involved in the living organism. Thus, to better understand the influence of the degradation on the implant integration and the failure of implants under mechanical load, a full characterization of the bone-to-implant interface is needed (i.e. biomechanically, morphologically, and biochemically). We report on the development of a push-out device to characterize the morphological and biomechanical properties of Mg-based implants under load conditions and report on the first successful in situ measurements.

\section{EXPERIMENTS}

In order to investigate the failure mechanisms of Mg-based implants, a load frame was developed which allows the acquisition of a sequence of tomographic data sets under compressive load conditions. Implants used were slotted grub screws made of two different extruded alloys of Mg and Gadolinium (Gd): Mg-5Gd and Mg-10Gd. Screws were $4 \mathrm{~mm}$ long, $2 \mathrm{~mm}$ in diameter, and had an M2 thread. As reference materials to compare with polyether ether ketone (PEEK) and Titanium were used. The screws were implanted in the femur of Sprague Dawley rats for various healing times $(4,8$, and 12 weeks). After the rats were sacrificed, cylindrical or box-shaped explants with a diameter/width of $5 \mathrm{~mm}$ were cut from the rat's femur. Tomograms were acquired from three different explants and two contrast modalities were compared. When imaging soft tissues or, more generally, objects with only small variations of their x-ray attenuation, conventional absorption tomography typically lacks contrast. In these cases phase-contrast techniques can be used which are sensitive to phase shifts induced in the transmitted wave front when X-rays propagate through the sample. In the following, we made use of conventional absorption contrast and differential phase contrast employing a grating interferometer.

\subsection{Tomography setup}

Data sets were acquired at the imaging beamline (IBL) P05 which is operated by the Helmholtz-Zentrum Geesthacht (HZG) at the PETRA III storage ring at the Deutsches Elektronen-Synchrotron (DESY) $)^{5,6,7}$. The X-ray source was an undulator insertion device located $80 \mathrm{~m}$ upstream the experimental stage. A double crystal monochromator (DCM) providing a relative bandwidth of $\Delta \mathrm{E} / \mathrm{E} \approx 10^{-4}$ was used. Imaging modalities employed were conventional absorption contrast and differential phase contrast (DPC) using a grating interferometer ${ }^{8,9}$. In addition, propagation-based phase contrast techniques $^{10,11}$ are available at the microtomography endstation of IBL. All tomograms were acquired at a photon energy of $34 \mathrm{keV}$ and using a step-wise rotation with 1200 projections. Images were acquired using an indirect detector system with a $\mathrm{CdWO}_{4}$-scintillator to convert X-rays into visible light which was magnified by microscope optics and detected by two different cameras. For absorption contrast, a CCD camera was used with 3056 x 3056 pixels, a linear pixel size of $12 \mu \mathrm{m}$, a dynamic range of 16-bit, and low noise, but with a slow readout. For phase-contrast, a CMOS camera was used with $5120 \times 3840$ pixels, a linear pixel size of $6.4 \mu \mathrm{m}$, a dynamic range of 12-bit, and a fast read out, but with higher noise compared to the CCD camera. The microscope optics was used at fivefold magnification. This resulted in an effective pixel size of $2.43 \mu \mathrm{m}$ and a horizontal field of view (FOV) of $7.3 \mathrm{~mm}$ for the CCD camera and an effective pixel size of $1.28 \mu \mathrm{m}$ and a horizontal FOV of $6.6 \mathrm{~mm}$ for the CMOS camera.

For differential phase-contrast, a grating interferometer ${ }^{8}$ with a single phase grating was used. The grating had a periodicity of $4.8 \mu \mathrm{m}$ and a structure height of $14.0 \mu \mathrm{m}$. In order to induce a phase shift of $\pi / 2$ at an energy of $34 \mathrm{keV}$, the distance between phase grating and scintillator was set to $15.5 \mathrm{~mm}$ and the grating was tilted towards the beam about 38.6 degrees. To resolve the interference pattern stemming from the phase grating and the sample, a stepping approach with three steps was employed. In combination with the CMOS camera, the effective pixel size is sufficient to render a second analyzer (absorption) grating unnecessary .

Because of the strong coherence of the X-ray beam, absorption-contrast data shows edge-enhancement as a result of the small, but finite propagation distance between sample and scintillator of $60 \mathrm{~mm}$ which is due to the extent of the sample environment. This can partially be accounted for by phase retrieval techniques such as the linearized version of the transport of intensity equation which is often referred to as Paganin phase retrieval. Acting as a low-pass filter on the 
recorded intensity maps, Paganin phase retrieval removes edge-enhancement fringes, suppresses noise, and enhances contrast at the expense of spatial resolution. The flat-and-dark-field corrected projections were typically $2 \times 2$ binned before tomographic reconstruction. For data processing and reconstruction, IDL and MATLAB routines were used. Tomographic reconstruction of absorption-contrast data was done with the ASTRA toolbox ${ }^{12,13}$.

\subsection{Load frame}

For mechanical testing, a load frame (see Figure 1) was developed for in situ experiments allowing the acquisition of a tomographic data set under static or cyclic load conditions. The load frame was designed to offer a highly flexible and modular system i.e. being deployable for a wide range of stresses, samples, and experimental conditions. The main components are a motorized actuator delivering forces up to $1 \mathrm{kN}$, various load cells with sensitivities for different force ranges, a signal amplifier for the load cells, and a cylindrical, X-ray transparent chamber acting as a spacer between base plate actuator and ensuring the force closure. The spacer is made of PEEK or, if only low forces are required, acrylic glass. Diameter and wall thickness of the spacer can be adapted to the envisaged forces or imaging modalities. For example, in our experiments, the wall thickness and diameter was chosen to withstand forces of at least $200 \mathrm{~N}$. In general, propagation-based phase contrast benefits from larger diameters in order to reduce phase variations stemming from horizontally varying optical path lengths through the spacer. The signal from the load cell is delivered to a signal amplifier via the slip ring of the tomography stage. The load frame can be operated in tension and compression for pushor pull-out experiments. In push-out experiments, the force is applied by the actuator onto the sample via an appropriately sized pin made of metal or PEEK. For hardness tests an indenter such as a Vickers tip can be mounted. Future add-ons will include the monitoring and the control of the humidity and temperature within the chamber. Control of the actuator, calibration of the load cell, and logging of the force signal is fully integrated in the beamline control software. The load frame and earlier prototypes of it have already been successfully employed in compression experiments on, e.g., bone-implants, mammalian teeth, and ivory (Vickers hardness test).

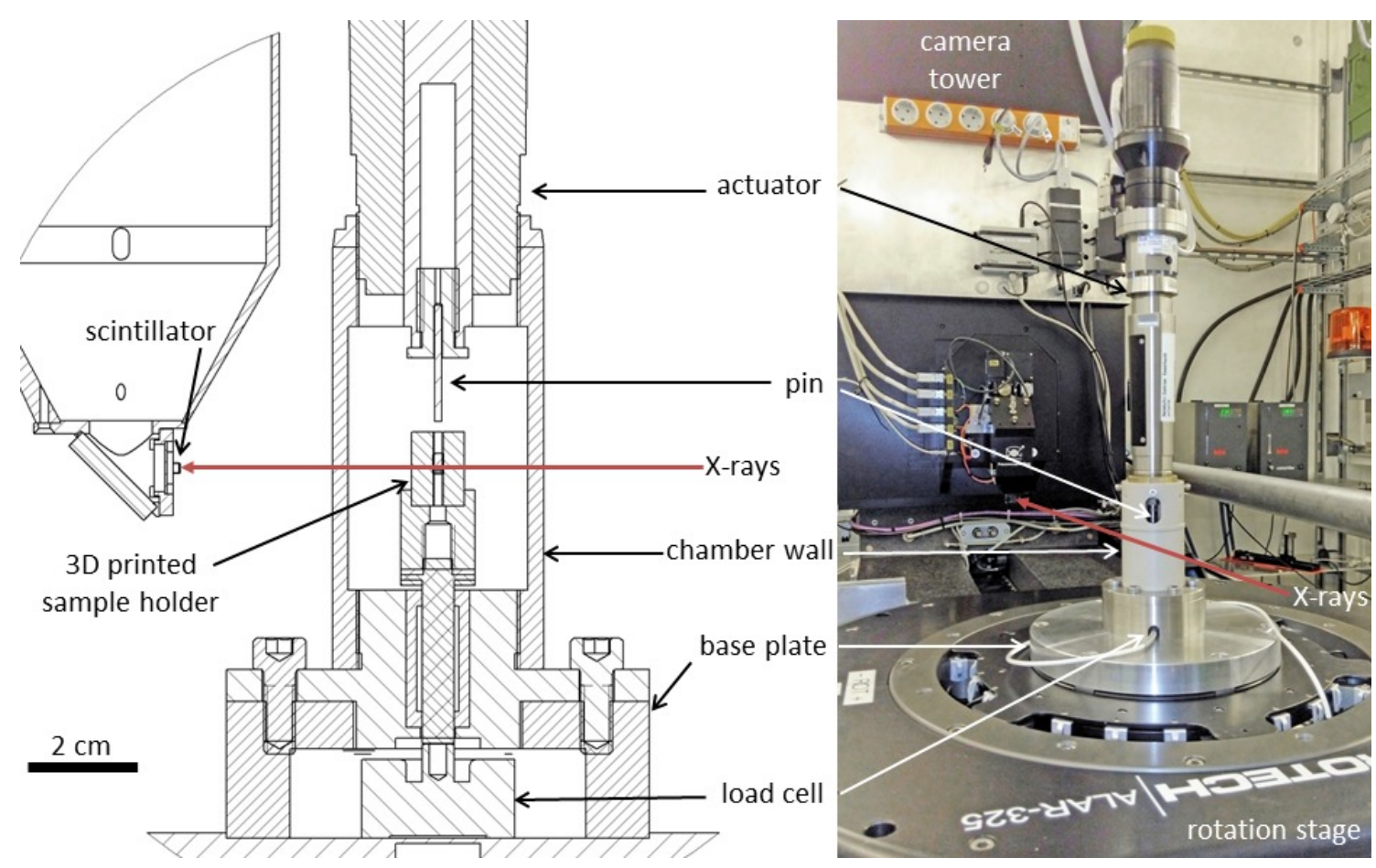

Figure 1: Left: Schematic drawing of the load frame. Right: Photograph of the load frame installed at the imaging beamline P05 at DESY. The load frame is mounted on top of the rotation axis. In the background, the camera tower is shown which contains a CMOS and a CCD camera. Attached to the front of the tower is the optical microscope with the scintillator where the X-rays are converted to visible light which is subsequently magnified and relayed onto the camera chip. 


\subsection{Sample mount}

In order to stabilize and to align the bone-implant sample such that the force is applied paraxially to the principal axis of the screw implant, customized 3D-printed sample holders were created. To do so, a fast scan of the sample is taken using a laboratory CT-scanner (Nanotom S, General Electric). After tomographic reconstruction, the screw is segmented using a connected threshold grower. The segmented screw is then used to reorientate the volume such that the principal axis of the screw is aligned vertically and centered within the volume. Then, the entire reoriented sample is segmented by simple thresholding. On the basis of this segmentation a cavity within a cylindrical volume is generated such that the sample can be inserted from above. From this cast, a 3D surface (STL) model is created which can be printed using commercially available $3 \mathrm{D}$ printers.

\subsection{Tomography under load conditions}

For the herein reported measurements, a sequence of ten tomograms of an explant was acquired under increasing load conditions and using conventional absorption tomography (see Figures 5 and 6). The sample used was an explant of a screw made of Mg-10Gd. The screw was implanted in rat femur for 12 weeks. The force applied onto the implant before the tomographic acquisition was successively increased from $0 \mathrm{~N}$ to $24 \mathrm{~N}$ in steps of $2.5 \mathrm{~N}$, see Figure 2 . After each increase in force, a series of radiographs was taken for about $20 \mathrm{~min}$ in order to wait for and to monitor relaxation processes. Then the acquisition of a tomogram was started. The force was monitored for each acquired projection. During tomography, a relaxation of the applied force of $0.5 \mathrm{~N}$ up to $4 \mathrm{~N}$ occurred, see Figure 2.

Because of temporary stability issues with the CMOS camera, tomograms using the DPC setup could only be acquired without load. Here we measured two explants which were mounted in the load frame. One implant was made of Mg-5Gd and the other of PEEK, see Figures 3 and 4, respectively.

\section{RESULTS \& DISCUSSION}

First we will report on the static measurements comparing differential phase contrast and adsorption contrast

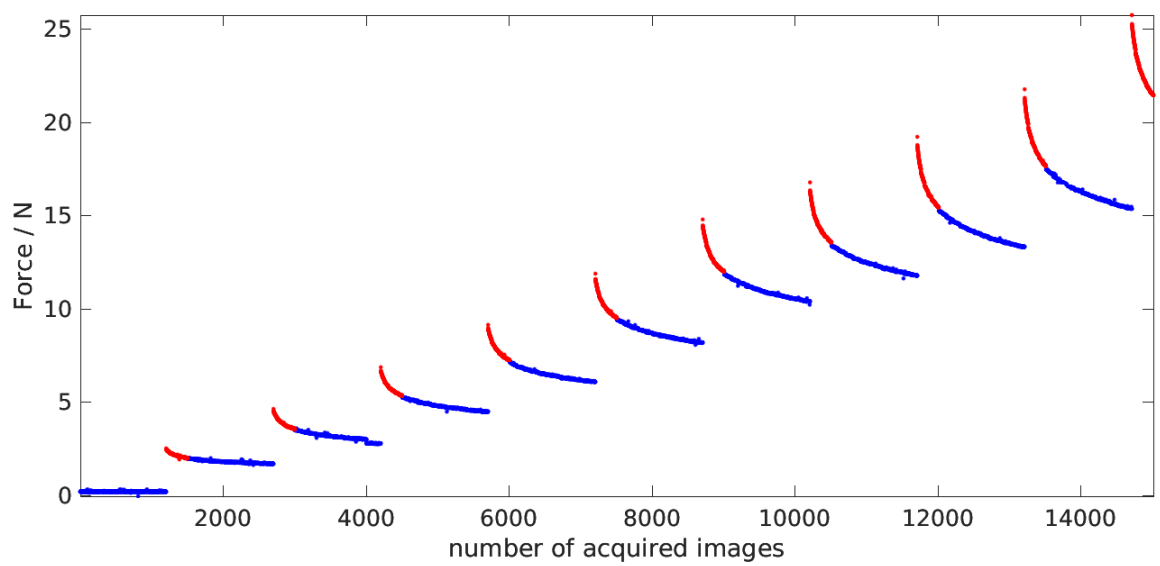

Figure 2: Force measured by the load cell during the sequence of tomography (blue dots) and radiography (red dots) of an explant under compressive load. After each tomogram, the actuator was moved until the applied force was increased by $2.5 \mathrm{~N}$ starting from $0 \mathrm{~N}$. After the force was set, 300 radiographs of the sample were taken to account for and monitor relaxation processes in the sample. Then a tomographic scan was acquired using 1200 projections. In total, a number of ten tomograms were taken. During tomography and radiography, a relaxation of the force occurred. This, however, rarely affected the data as only small and localized movement artefacts are present in the tomographic reconstructions. 


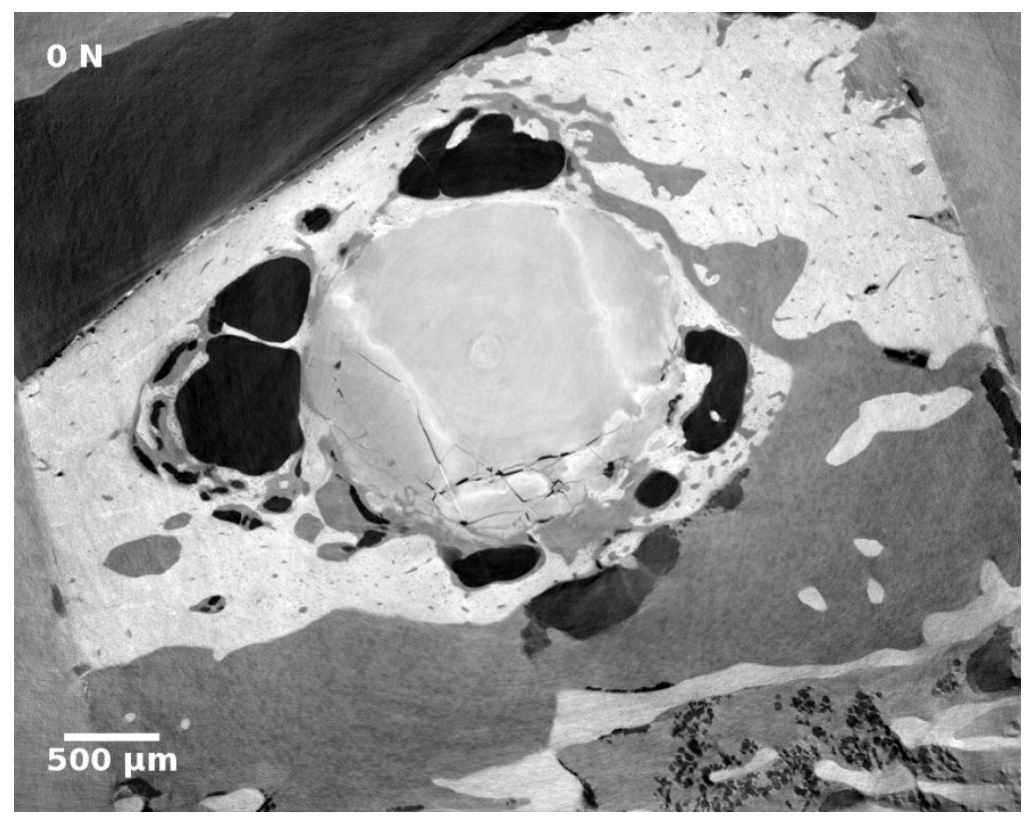

Figure 3: Differential phase contrast tomography of an Mg-5Gd screw implanted in a rat femur after 12 weeks of healing. The image shows a horizontal slice through the tomographic reconstruction of an explant mounted in the load frame at $0 \mathrm{~N}$. The depicted slice was $2 \times 2$ binned after reconstruction and has a shape of $1024 \times 812$ pixels and a linear pixel size of $5.12 \mu \mathrm{m}$ after binning.

measurements to quantify which contrast mechanism is better suited for this kind of samples. In differential-phasecontrast mode employing a single grating, tomography of two explants mounted in the load frame was performed: one screw made of $\mathrm{Mg}-5 \mathrm{Gd}$ and implanted for 12 weeks before being harvested and one screw made of PEEK where the rat died after implantation. The tomographic reconstructions exhibit a good density contrast. Moreover, fringes due to edgeenhancement are absent in contrast to the tomograms acquired with conventional absorption contrast, compare Figures 3 and 4 with Figures 5 and 6 . In the latter case, the small but finite distance between sample and scintillator, which is due to the chamber wall, was sufficient to give rise to fringes in the tomographic reconstructions, e.g., consider the boundary of the bone in Figures 5 and 6.

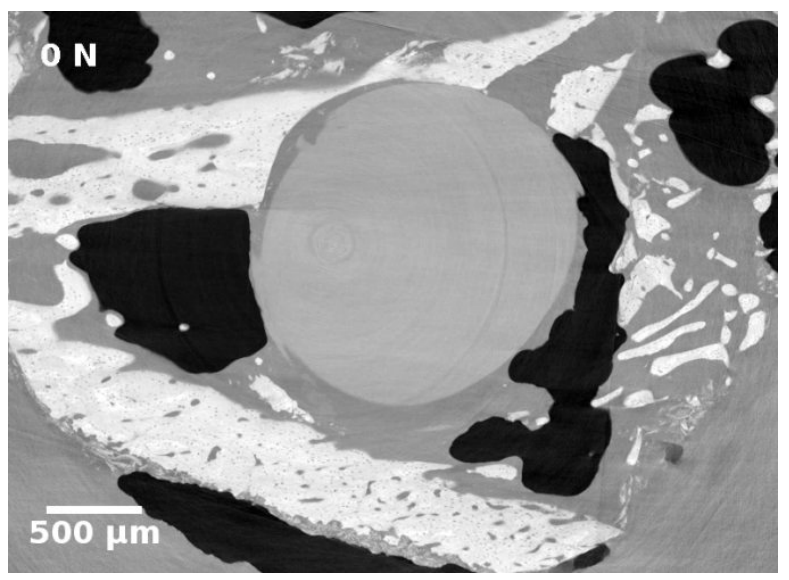

Figure 4: Differential phase contrast tomography of a PEEK screw implanted in a rat femur. The image shows a horizontal slice through the tomographic reconstruction of an explant mounted in the load frame at $0 \mathrm{~N}$. The rat died shortly after implantation and no bone growth took place. The depicted slice was $2 \times 2$ binned after reconstruction and has a shape of $782 \times 570$ pixels and a linear pixel size of $5.12 \mu \mathrm{m}$ after binning. 
In the following we will report on the first push-out test of an explant. Using conventional absorption tomography, a sequence of ten tomograms under increasing compressive load conditions was acquired. Between scans the force maximum applied onto the screw was increased from $0 \mathrm{~N}$ up to $24 \mathrm{~N}$ in steps of $2.5 \mathrm{~N}$. The screw was made of Mg- $10 \mathrm{Gd}$ and was implanted in a rat femur for 12 weeks before being harvested. The relaxation of the applied force as described in section 2.4 rarely affected the tomographic reconstruction as artefacts related to sample movement are locally restricted
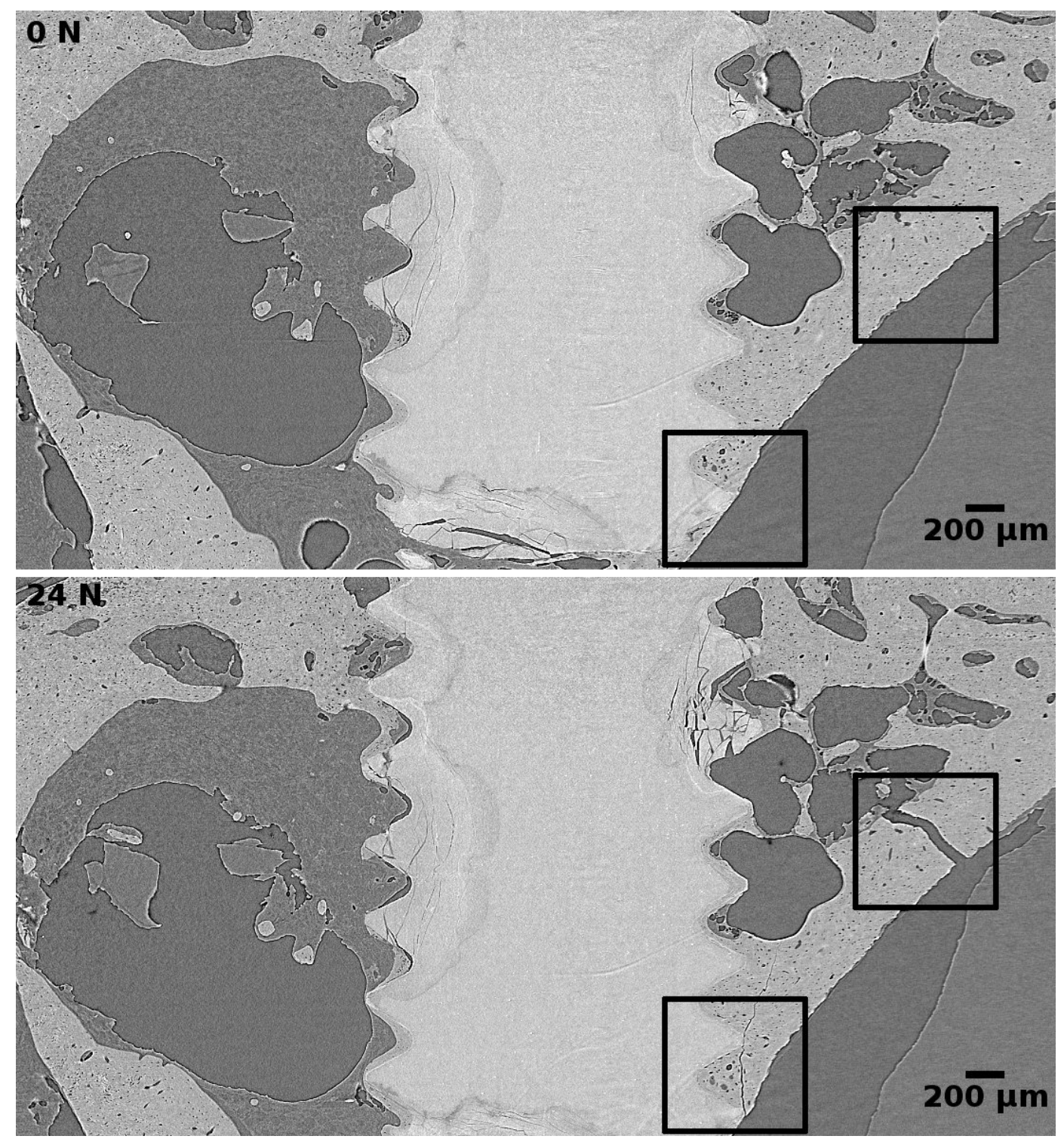

Figure 5: Vertical slices through the tomographic reconstructions of an explant imaged without load (top) and under mechanical load (bottom). Images show an explant of a Mg-10Gd screw in a rat femur 12 weeks after implantation. Different corrosion layers between implant and bone are clearly distinguishable. The bottom tomogram was acquired under mechanical load where a force of $24 \mathrm{~N}$ was applied before the image acquisition started. Breakage is clearly visible throughout the sample. The black rectangles indicate two regions where rupture occurs and which are depicted in the load sequence in Figure 6. Absorption contrast tomography was used for the load sequence. The depicted slices have a shape of 1096 x 592 pixels with a linear pixel size of 4.8 $\mu \mathrm{m}$. 
and relatively small, see Figures 5 and 6. Vertical slices through the tomographic reconstructions are shown in Figure 5. The top image in Figure 5 was acquired without load while the bottom image was acquired after a maximum force of 24 $\mathrm{N}$ was applied. The bottom image in Figure 5 and the image sequences in Figure 6 clearly show breakage throughout the sample which was induced by the applied load. The initial fragmentation of the implant material in the areas where corrosion took place, as can be seen in Figures 3 and 5, is not due to load. This is a normal consequence of corrosion and can also be observed in corroded areas of screws that are not implanted but which have been immersed in a cell culture medium for one week or longer. However, cracks in these fragmented areas increase under increasing load. Note that the interface between implant material and bone does not exhibit a well-defined boundary surface as there is always a corrosion layer in between. Moreover, the corrosion layer is not homogeneous and the composition of which can influenced by the bone. Considering the image sequence of the magnified regions in Figure 6, lines/planes of breakage and the propagation of which do not occur directly at the bone-to-implant interface indicating a strong connection and integration of the implant. These preliminary findings support the choice of Mg-based alloys as candidates for implant material.

Either of the employed contrast modalities allows distinguishing the remaining core material of the magnesium-based screws, different layers of corrosion, cortical bone with osteocytes and Haversian canals within, and bone marrow.

\section{CONLUSION}

A load frame for in-situ tomography experiments under load conditions was developed at the imaging beamline P05 at PETRA III at DESY. In a first push-out experiment, a series of tomograms of a bone-implant sample under increasing load conditions was acquired. The reconstructed volumes demonstrate a good image quality and a high spatial resolution that allows to resolve and to distinguish cortical and trabecular bone, osteocytes lacunae, Haversian canals, and bone marrow. Comparing the employed contrast modalities at the given spatial resolution, differential phase contrast is superior to absorption contrast in the sense that the contrast in the tomographic reconstruction is improved and fringes due to edge enhancement are absent. The analyzed load sequence showed that the bone-to-implant interface remained intact under compressive forces of up to about $18 \mathrm{~N}$. When failure took place within the sample, sites of fracture occurred throughout the sample. However, breakage does not seem to predominantly originate from or propagate along
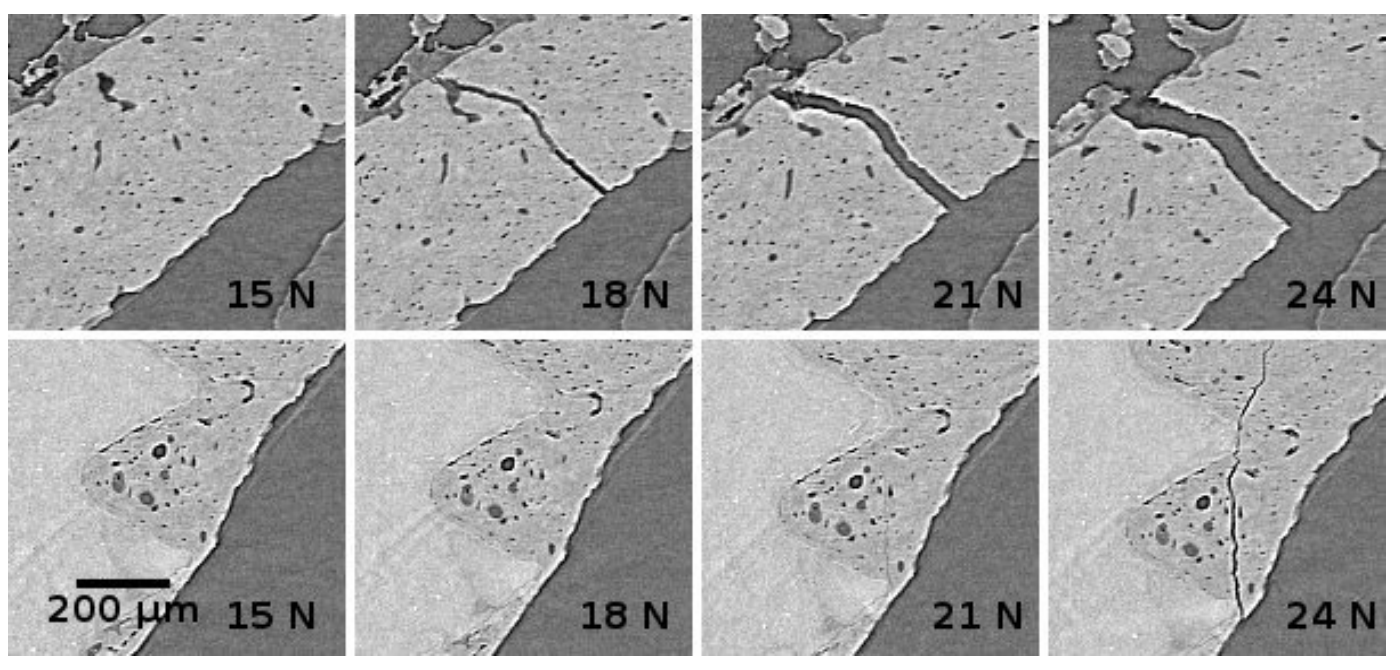

Figure 6: Sequences of vertical slices through the tomographic reconstructions of an explant under increasing compressive load. The explant is a Mg-10Gd screw in a rat femur 12 weeks after implantation. The depicted images are magnified details of the regions indicated in Figure 5. The top row depicts a region of cortical bone as indicated by the top right rectangle in Figure 5. The bottom row depicts a region of the bone-to-implant interface as indicated by the bottom left rectangle in Figure 5. Before the acquisition of the tomograms the force was set to $15 \mathrm{~N}, 18 \mathrm{~N}, 21 \mathrm{~N}$, and $24 \mathrm{~N}$, respectively. Each region of interest has a shape of $148 \times 140$ pixels with a linear pixel size of $4.8 \mu \mathrm{m}$. 
the corroded areas at the bone-to-implant interface. Cracks of the fragmented areas of the corroded screw, which are already present prior to load, increase under load. But this does not seem to impair the stability of the implant as in these regions lines/planes of breakage which are due to load are not more frequent than in other regions. Moreover, the analyzed Mg-based implants exhibit a tight integration into the bone. The data acquired in this first proof-of-concept experiment suggests that screws made of Mg-10Gd are candidates well suited for bone implants. In follow-up experiments a statistically significant number of implants will be considered which will give evidence on the performance of the employed implant material i.e. on integration and stability with respect to the employed material, healing times, and load conditions.

\section{ACKNOWLEDGMENTS}

This research was carried out within the SynchroLoad project (BMBF project number 05K16CGA) and the MgBone project (BMBF project number 05K16CGB) which are funded by the Röntgen-Ångström Cluster (RÅC), a bilateral research collaboration of the Swedish government and the German Federal Ministry of Education and Research (BMBF). We acknowledge provision of beamtime, related to the proposals I-20160658 and I-20160104, at beamline P05 at PETRA III at DESY, a member of the Helmholtz Association (HGF). For the development of the 20 MPixel CMOS detector within the Helmholtz project Detector Technology and Systems Platform (DTS) we like to thank Michele Caselle, Matthias Vogelgesang, and Andreas Kopmann of the Institute for Data Processing and Electronics of the Karlsruhe Institute of Technology, Germany.

\section{REFERENCES}

[1] S. H. Kim, J. H. Oh, O.-S. Lee, H.-R. Lee, and A. R. Hargens, "Postoperative imaging of bioabsorbable anchors in rotator cuff repair," The American Journal of Sports Medicine, vol. 42, no. 3, pp. 552-557, 2014, pMID: 24431337. [Online]. Available: http://dx.doi.org/10.1177/0363546513517538

[2] R. Mittal, J. Morley, H. Dinopoulos, E. G. Drakoulakis, E. Vermani, and P. V. Giannoudis, "Use of bioresorbable implants for stabilisation of distal radius fractures: the United Kingdom patients' perspective," Injury, vol. 36, no. 2, pp. 333-338, 2005. [Online]. Available: http://dx.doi.org/10.1016/j.injury.2004.09.015

[3] B. J. Luthringer, F. Feyerabend, and R. Willumeit-Römer, "Magnesium-based implants: a mini-review," Magnes Res, vol. 27, no. 4, pp. 142-154, 2014.

[4] S. Virtanen, "Biodegradable $\mathrm{mg}$ and $\mathrm{mg}$ alloys: Corrosion and biocompatibility," Materials Science and Engineering: B, vol. 176, no. 20, pp. 1600 - 1608, 2011, 2nd Symposium on Biodegradable Metals. [Online]. Available: http://www.sciencedirect.com/science/article/pii/S0921510711002364

[5] A. Haibel, F. Beckmann, T. Dose, J. Herzen, M. Ogurreck, M. Müller, and A. Schreyer, "Latest developments in microtomography and nanotomography at PETRA III," Powder Diffraction, vol. 25, no. 2, p. 161-164, 2010.

[6] A. Haibel, M. Ogurreck, F. Beckmann, T. Dose, F. Wilde, J. Herzen, M. Müller, A. Schreyer, V. Nazmov, M. Simon, A. Last, and J. Mohr, "Micro- and nano-tomography at the GKSS imaging beamline at PETRA III," pp. 78040B-78040B-8, 2010. [Online]. Available: http://dx.doi.org/10.1117/12.860852

[7] F. Wilde, M. Ogurreck, I. Greving, J. U. Hammel, F. Beckmann, A. Hipp, L. Lottermoser, I. Khokhriakov, P. Lytaev, T. Dose, H. Burmester, M. Müller, and A. Schreyer, "Micro-CT at the imaging beamline P05 at PETRA III," AIP Conference Proceedings, vol. 1741, no. 1, p. 030035, 2016. [Online]. Available: http://aip.scitation.org/doi/abs/$10.1063 / 1.4952858$

[8] A. Hipp, F. Beckmann, P. Lytaev, I. Greving, L. Lottermoser, T. Dose, R. Kirchhof, H. Burmester, A. Schreyer, and J. Herzen, "Grating-based x-ray phase-contrast imaging at PETRA III," pp. 921206-921206-12, 2014. [Online]. Available: http://dx.doi.org/10.1117/12.2061776

[9] A. Momose, S. Kawamoto, I. Koyama, Y. Hamaishi, K. Takai, and Y. Suzuki, "Demonstration of X-ray Talbot interferometry," Japanese Journal of Applied Physics, vol. 42, no. 7B, pp. L 866-L 868, 2003. [Online]. Available: http://stacks.iop.org/1347-4065/42/i=7B/a=L866

[10] A. Snigirev, I. Snigireva, V. Kohn, S. Kuznetsov, and I. Schelokov, "On the possibilities of x-ray phase contrast microimaging by coherent high-energy synchrotron radiation,” Rev. Sci. Instrum., vol. 66, no. 12, pp. 5486-5492, 1995. [Online]. Available: http://link.aip.org/link/?RSI/66/5486/1

[11] S. W. Wilkins, T. E. Gureyev, D. Gao, A. Pogany, and A. W. Stevenson, "Phase-contrast imaging using polychromatic hard X-rays," Nature, vol. 384, no. 6607, pp. 335-338, Nov. 1996. [Online]. Available: http://dx.doi.org/$10.1038 / 384335 \mathrm{a} 0$ 
[12] W. Palenstijn, K. Batenburg, and J. Sijbers, "Performance improvements for iterative electron tomography reconstruction using graphics processing units (GPUs)," Journal of Structural Biology, vol. 176, no. 2, pp. 250 - 253, 2011. [Online]. Available: http://www.sciencedirect.com/science/article/pii/S1047847711002267

[13] W. van Aarle, W. J. Palenstijn, J. D. Beenhouwer, T. Altantzis, S. Bals, K. J. Batenburg, and J. Sijbers, "The ASTRA toolbox: A platform for advanced algorithm development in electron tomography," Ultramicroscopy, vol. 157, no. 0, pp. 35 - 47, 2015. [Online]. Available: http://www.sciencedirect.com/science/article/pii/S0304399115001060 